\title{
Electrophysiological Parameters of Sinus Node Function in Patients with Paroxysmal Tachyarrhythmias
}

\author{
Kotlyarov A.A. ${ }^{1}$, Kokorev A.V. ${ }^{1}$, Balykova L.A. ${ }^{2}$, Pyataev N.A. ${ }^{2}$, Moiseeva I.Y. ${ }^{3}$, \\ and Kotlyarova Y.A. ${ }^{1}$ \\ ${ }^{1}$ Obninsk Institute for Nuclear Power Engineering of the National Research Nuclear University \\ MEPhl, Studgorodok 1, Obninsk, Kaluga region, 249040, Russia \\ ${ }^{2}$ N.P.Ogarev Mordovia State University, Saransk, Russia \\ 3 Penza State University, Russia
}

\section{Abstract}

to analyze the indicators of the function of the sinus node in patients of young age with paroxysmal tachycardia. Methods: study included 11 patients with suspected paroxysmal tachycardia, with an average age of $17 \pm 28$. The basis for holding transesophageal electrophysiological study (TE EPS) was the clinical and

Corresponding Author: Andrey Kotlyarov AAKotlyarov@mephi.ru

Received: 17 January 2018 Accepted: 25 March 2018 Published: 17 April 2018

Publishing services provided by Knowledge $\mathrm{E}$

(c) Kotlyarov A.A. et al. This article is distributed under the terms of the

Attribution License, which permits unrestricted use and redistribution provided that the original author and source are credited.

Selection and Peer-review under the responsibility of the PhysBioSymp17 Conference Committee. electrophysiological characteristics of paroxysmal tachycardia. According to the results of Holter monitoring ECG (HM ECG) analyzed the minimum and maximum heart rate, number of ventricular and supraventricular arrhythmias, presence of pauses, rhythm and episodes of paroxysmal tachycardia. According to CHP, EFI estimated the initial heart rate (HR), recovery time of sinus node function (RTSNF), corrected recovery time of sinus node function (CRTSNF), point of Wenkebach ( $p$. W), duration of the effective refractory period of the atrioventricular connections, presence of aberrant complexes and episodes of paroxysmal tachycardias before and after administration of atropine at a dose of $0.02 \mathrm{mg} / \mathrm{kg}$. Results: Complaints characteristic of the tachyarrhythmia was diagnosed in 9 patients, episodes of heart rate more than 150 beats per minute in 7 patients. When conducting TE EPS obtained the following results: episodes of supraventricular tachycardia provoked in 8 patients (in two cases of paroxysmal tachycardia managed to provoke only after administration of atropine). Three of them have shimmer and atrial flutter episodes reciprocal tachycardia in five. Three patients provoke paroxysmal tachycardia failed, but they showed a shortening of the PQ interval and the appearance of aberrant QRS complexes when stimulated. In patients with paroxysmal SVT signs of sinus node dysfunction was detected in 6 patients, in the form of episodes of sinus arrhythmia (4 patients), migration pacemaker the atria (4 patients), sinoatrial blockade of II degree (3 patients), blockade of legs of bunch of gisa (2 patients), atrioventricular block degree II-III (1 patient), RTSNF more than 1500 MS in 1 patient, CRTSNF greater than 500 msec in 3 patients. Conclusion: in 6 of 9 patients with supraventricular paroxysmal tachycardia revealed signs of sinus node dysfunction, probably has a vagotonic in nature. 


\section{Relevance}

Patients of young age quite often have disturbances of a rhythm and conductivity which are risk factors of sudden death. The clinical picture can be various, is more often displays of arrhythmias are: dysfunction of a sinus node with a syndrome takhi-and bradyarrhythmias or preexcitement of ventricles in the presence of additional ways of atrioventricular carrying out. The listed changes can proceed asymptomatically, or not be followed by accurately outlined complaints in this connection, they it is long remain not distinguished. At difficulty of diagnosis is necessary to conduct a transesophageal electrophysiologic, to clarify the nature of arrhythmias.

\section{Work purpose}

to analyze indications to carrying out and results of a transesophageal electrophysiologic research at patients of young age.

\section{Methods}

The research included 22 patients, aged from 17 up to 28 years. All patients were divided into 2 groups depending on the preliminary diagnosis. The first group $(n=11)$ was made by patients with dysfunction of a sinus node and a sick sinus syndrome; the second group $(n=11)$ patients with Bouveret's diseases and a WPW-syndrome (WPW).

The subjective condition of patients was estimated by the number of complaints. Considered complaints on: dizziness, feeling of heart beat, an asthma, interruptions in cardiac performance, pain in heart, an infrequent pulse, weakness, losses of consciousness, preunconscious states.

The program of inspection included carrying out an electrocardiography, holter monitoring and a transesophageal electrophysiologic research. By results of an electrocardiography estimated (the provision of an electrical axis of heart, the minimum and maximum ChSS, a pacemaker, $\mathrm{PQ}$ interval duration). By results of holter monitoring analyzed (the minimum and maximum ChSS, the number of ventricular and supraventricular disturbances of a heart rhythm, a sinus arrhythmia, existence of episodes of an asystolia lasting 1,5 sec. and more and episodes of a Bouveret's disease, existence of $C A$ and $A B$ of heart blocks). By results of a transesophageal electrophysiologic research. estimated (VVFSU, KVVFSU, t. In, duration of ERP of an atrioventricular node, 
existence of aberrant complexes and episodes of a Bouveret's disease before administration of atropine in a dose of $0.02 \mathrm{mg} / \mathrm{kg}$ ). Repeated determination of parameters carried out after intravenous administration $0.1 \%$ of solution of atropine in a dose of a component of $0.02 \mathrm{mg} / \mathrm{kg}$.

Statistical processing of results of a research was carried out by means of a package of the statistical Excel 2002 programs. Mathematical processing included calculations of arithmetic averages of values $(M)$, their mistakes $(m)$, reliability of distinction of arithmetic averages $(R)$ by means of Styyudent's t-criterion.

\section{Results}

The short characteristic of patients is presented in tab. 1.

TABLE 1: Characteristic of patients.

\begin{tabular}{|c|c|c|c|c|c|c|}
\hline & Quantity & M/ZH & Age & DSU/SSSU & FRIDAY & WPW syndrome \\
\hline I group & 11 & 11 & $19 \pm 0.7$ & 7 & 2 & 1 \\
\hline II group & 11 & $9 / 2$ & $20 \pm 0.7$ & 5 & 4 & 6 \\
\hline
\end{tabular}

Patients showed various complaints caused by the analyzed pathology (dizziness, a heart consciousness, an asthma, interruptions in cardiac performance, pain in heart, an infrequent pulse, the expressed weakness, a loss of consciousness, preunconscious states).

To all patients for specification of nature of disturbance of a heart rhythm electrocardiographic examination was conducted. Estimated the minimum and maximum ChSS, a pacemaker, duration of the main intervals, results are presented in tab. 2.

TABLE 2: Values of the ECG parameters at patients.

\begin{tabular}{l|c|c|c|c|} 
Patients & Chssmin & Chssmax & MBP & PQ shortening \\
\hline I group & $61.1 \pm 6$ & $76 \pm 10$ & $43 \pm 5 \cdot 4-74.3 \pm 2.1$ & $1(9 \%)$ \\
\hline II group & $62.6 \pm 5.1$ & $79 \pm 6.3$ & $51.1 \pm 15.5-124 \pm 27.1$ & $3(27 \%)$ \\
\hline
\end{tabular} $\begin{aligned} & \text { Designation: ChSS min - heart rate minimum; ChSS max - heart rate maximum; } \\
& \text { MVR - migration of a pacemaker. }\end{aligned}$

To patients daily monitoring of an electrocardiography is executed. At patients of the first ChSS min group made 35 \pm 1.2 , ChSS max 153 \pm 7.3 , in the second ChSS min $39.4 \pm 0.9$, ChSS max $162 \pm 4.2$ group. SA episodes in the first group made $15 \pm 7$, in the second group $7 \pm 1$. The quantity of episodes of an asystolia lasting more than $1.5 \mathrm{sec}$. in the first group 
$1.65 \pm 0.2$ in days, in the second group of episodes of an asystolia was not observed (tab. 3).

TABLE 3: Results of HM-EKG.

\begin{tabular}{|c|c|c|c|c|c|c|}
\hline Patients & $\begin{array}{c}\text { Chssmin, in } \\
\text { min. }\end{array}$ & $\begin{array}{c}\text { Chssmax, in } \\
\text { min. }\end{array}$ & $\begin{array}{c}\text { SA blockade, } \\
\text { episodes in } \\
\text { min. }\end{array}$ & $\begin{array}{c}\text { Quantity of } \\
\text { episodes of } \\
\text { an asystolia } \\
\text { more than } 1,5 \\
\text { sec./days }\end{array}$ & NZHE & ZHE \\
\hline I group & $35 \pm 1.2$ & $153 \pm 7.3$ & $15 \pm 7$ & $1.65 \pm 0,2$ & $64.4 \pm 23.8$ & $1 \pm 0$ \\
\hline II group & $39.4 \pm 0.9$ & $162 \pm 4.2$ & $7 \pm 1$ & - & $27.4 \pm 14.9$ & $149.5 \pm 207$ \\
\hline
\end{tabular}

When carrying out daily monitoring of an ECG supraventricular and ventricular extrasystoles were revealed. The quantity of supraventricular extrasystoles in the first group made per day $64.4 \pm 23.8$, in the second group $27.4 \pm 14.9$; ventricular extrasystoles per day in the first group $-1 \pm 0$, in the second group $-149.5 \pm 207$. Reliable distinctions by quantity of extrasystoles in groups were not revealed.

In the first group ventricular extrasystoles are revealed at 2 patients, supraventricular at 9 patients. In the second group ventricular extrasystoles are also revealed at 2 patients, supraventricular at 5 patients.

When carrying out State of emergency of EFI determined VVFSU, KVVFSU, by t. In, ERP before administration of atropine in a dose of $0.02 \mathrm{mg} / \mathrm{kg}$.

In the first VVFSU group before administration of atropine there were on average $1342 \pm 84 \mathrm{~ms}$, and after introduction decreased to $694 \pm 87(p<0.001)$, in the second VVFSU group before administration of atropine 1093 \pm 94 , after introduction this indicator authentically decreased to $694 \pm 89 \mathrm{~ms}$ ( $p<0.02$ ). VVFSU more than $1500 \mathrm{~ms}$ is revealed at 4 patients (36\%) of the first group and at 1 patient $(9 \%)$ of the second group. KVVFSU in the first group before administration of atropine averaged $496 \pm 45$ $\mathrm{ms}$, after introduction of $194 \pm 26 \mathrm{~ms}(\mathrm{p}<0.001)$, in the second group before introduction of $374 \pm 50 \mathrm{~ms}$, after administration of atropine the indicator authentically decreased A0159 $\pm 66 \mathrm{~ms}(p<0.02)$. KVVFSU more than $500 \mathrm{~ms}$ in the first group is revealed at 6 patients (54\%) and at 3 patients in the second group is (tab. 4 ).

T. V. in the first group before administration of atropine was on average $156 \pm 12 \mathrm{~ms}$, after introduction this indicator authentically increased to $197 \pm 9 \mathrm{~ms}(p<0.02)$, in the second group before introduction $179 \pm 11 \mathrm{MC}$, after introduction of $210 \pm 10 \mathrm{~ms}$. T.V. of less than 140 in $1 \mathrm{~min}$. is revealed at 3 patients ( $27 \%$ ) in the first group and at 1 patient ( $9 \%)$ in the second group. ERP in the first group before administration of atropine averaged 
$310 \pm 47 \mathrm{~ms}$, after introduction of values it is not received, in the second group before introduction of $347 \pm 46 \mathrm{~ms}$, after introduction this indicator authentically decreased to $302 \pm 26 \mathrm{~ms}$. The number of patients with the tachycardia paroxysms (TP) in the first group made 2 patients (18\%) and at 4 patients (36\%) in the second group. Average ChSS at FRIDAY in the first group made $156 \pm 8.4 \mathrm{~B}$ mines, in the second group $185 \pm 17.3$ in min. (tab. 4).

TABLE 4: Values of parameters at patients when carrying out ChpEFI.

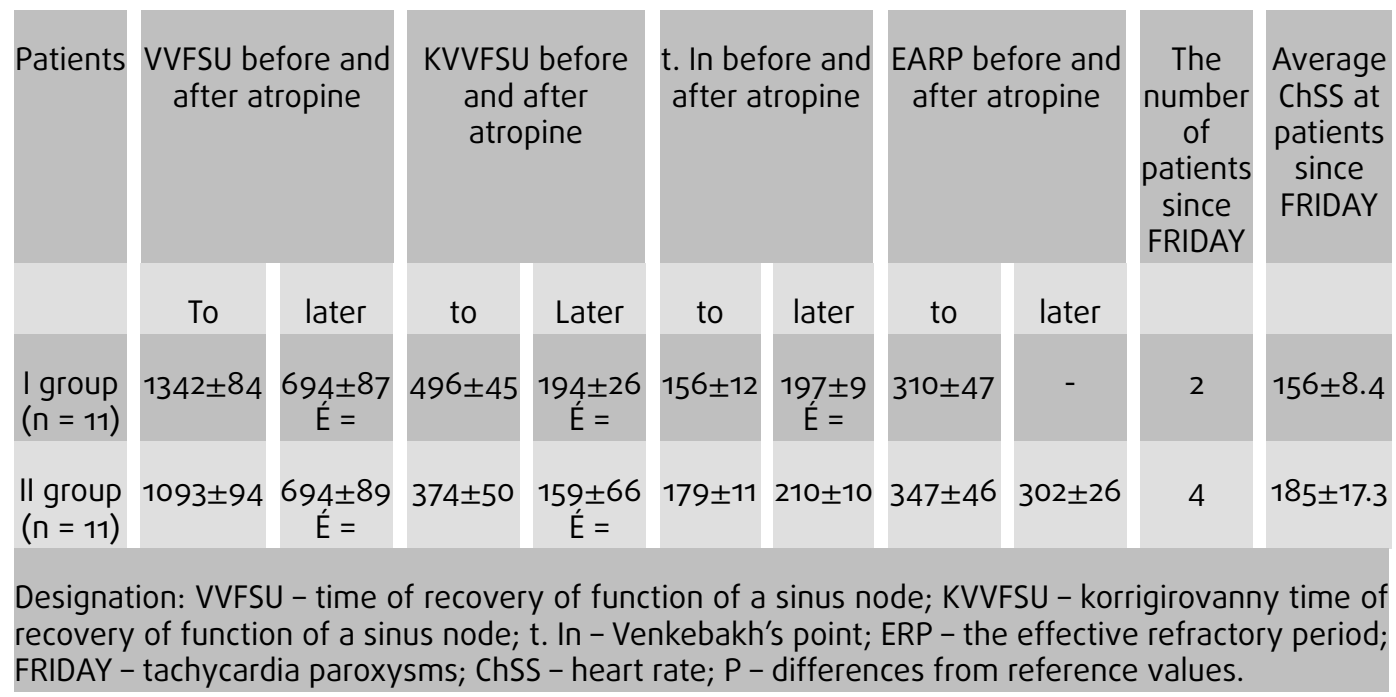

It is more patients lasting VVFSU more than $1500 \mathrm{~ms}$ in the first group (in the first group - 4 patients, in the second - at 1), it is also more than 500 ms of patients with KVVFSU more in the first group (6 - in the first group, 3 - in second), t. In less than 140 1 minute it is revealed at 3 patients in the I group, at 2 - in II). In the second group there are more patients with Bouveret's diseases (in the first group 2 patients, in second 4 patient) (fig. 4,5).

In the first group at 2 patients at superfrequent electrostimulation the steady paroxysm of a ciliary arrhythmia which was independently stopped is induced. In the second group the number of patients with the induced Bouveret's disease 4 . From them at 2 patients the paroxysm of reciprocal orthodromic supraventricular tachycardia stopped by superfrequent electrostimulation, at one patient at superfrequent electrostimulation is provoked the short episode of a ciliary arrhythmia and antidromic tachycardia which was independently stopped is provoked. At one patient the paroxysm of a ciliary arrhythmia which was independently stopped is induced. 


\section{Conclusion}

In the first group at 7 people symptoms of dysfunction of a sinus node (a vagotonia, a sick sinus syndrome) are revealed what was shown by bradycardia episodes (less than 40 beats per minute), existence of sinuatrial blockade, a sinus arrhythmia, migration of pacemakers, increase in time of recovery of function of a sinus node it is (more than 1500), korrigirovanny time of recovery of function of a sinus node (more than 500). Inadequate increase in ChSS after administration of atropine was observed at 2 patients. So, symptoms of dysfunction of a sinus node are revealed at 9 patients from 11. At two patients paroxysms of blinking and an atrial flutter with a frequency more than 160 a minute are provoked, at one patient signs of additional ways of carrying out were revealed that it was shown by shortening of an interval of $P Q$, emergence at stimulation of the aberrant QRS complexes.

In the II group episodes of a tachyarrhythmia are provoked at 4 patients. At one blinking and an atrial flutter, episodes of reciprocal tachycardia at three. To six patients, it was not succeeded to provoke a Bouveret's disease. But they recorded shortening of an interval of $P Q$ and emergence of the aberrant $Q R S$ complexes at stimulation. At five patients, signs of the vagotonia and dysfunction of a sinus node shown by episodes of bradycardia are revealed it is less than 40 beats per minute, emergence of sinuatrial blockade and an atrioventricular block, episodes of a sinus arrhythmia and migration of a pacemaker (by results of holter monitoring of the electrocardiogram), increase in korrigirovanny time of recovery of function of a sinus node more than $500 \mathrm{~ms}$. In two cases the Bouveret's disease managed to be provoked only after administration of atropine, in a dose of $0.02 \mathrm{mg} / \mathrm{kg}$.

Thus, at patients with suspicion of Bouveret's supraventricular disease dysfunctions of a sinus node that, perhaps, demonstrates development of a compensatory vagotonia as protective mechanism from a Bouveret's disease are revealed.

\section{References}

[1] Bogachyov M. I. New approach to the differential diagnosis of syncopal states / M. I. Bogachyov, I. S. Kireenkov, E. M. Nifontov, S. A. Pyko//Messenger of arrhythmology. -2008. -№52. - Page 50-56.

[2] Dzhanashiya P. H. Sick sinus syndrome / P.Kh. Dzhanashiya, N. M. Shevchenko, N. D. Dzhanashiya// http://www.rusmg.ru/php/content.php?id=352. (in russian) 
[3] Clinical recommendations about carrying out electrophysiologic researches, catheter ablation and use of the implanted antiarrhytmic devices. New edition 2009. Moscow. 303 pages. (in russian)

[4] Masur N. A. Sudden death (stratification of risk and prevention).//N. A. Masur. Heart. -2006. -№1. - Page. (in russian)

[5] Dysfunction and sick sinus syndrome//Cardiology. - 2007. - No. 2. - Page 5-11. (in russian)

[6] Primary (genetically determined) diseases of the carrying-out system сердца.//V. A. Shulman, S. Yu. Nikulina, G. Matyushin, Yu.Ivanitskaya. http: //www . cardiosite . $\mathrm{ru} /$ articles/article.asp?id=873\&keywords= . (in russian)

[7] Gallogher J.J., Smith W.M., Kerr C.R. et al. Esophageal pacing: diagnostic and therapeutic tool.//Circulation. - 2006. - Vol. 65. - N 2. - P. 336-341.

[8] Sud S, Klein GJ, Skanes AC, Gula LJ, Yee R, Krahn AD. Implications of mechanism of bradycardia on response to pacing in patients with unexplained syncope. Europace 2007; 9:312-318. 